\title{
Editorial: Special Issue on "Advanced Technologies and Practices in Recent Wireless Sensor Networks"
}

\author{
Taeshik Shon • Ching-Hsien Hsu • Liudong Xing
}

Published online: 21 April 2011

(C) Springer Science+Business Media, LLC. 2011

The recent advancement of wireless sensor network technologies and practices is one of the major roles to the evolution and enrichment of human life and fun with the advent of the next generation convergence network environments. A variety of usages and paradigms for wireless sensor networks is a growing interest in the design, development and deployment of sensor systems for applications with other emerging technologies such as RFID Technology, Multimedia Based Surveillance System, Biomedical Technology, Mobile Agent based Networks, P2P technology and Business Process, etc. In addition, the next evolutionary step for wireless sensor networks is to consider mobility, energy harvesting, and converged and fused services and applications.

Therefore, this special issue on advanced technologies and practices of recent wireless sensor networks presented current research focusing on MAC, security-related protocols and applications, robust architecture and frameworks, along with new theories and methodologies for next generation wireless sensor networks and respective its smart devices.

We received forty three manuscripts. The fourteen manuscripts were finally selected for this Special Issue after the first, second review processes. Each manuscript selected was blindly reviewed by at least three reviewers consisting of guest editors and external reviewers.

\footnotetext{
T. Shon $(\varangle)$

Division of Information and Computer Engineering, College of Information Technology, Ajou University, Suwon 443-749, Korea e-mail: taeshik.shon@gmail.com

C.-H. Hsu

Department of Computer Science and Information Engineering, Chung Hua University, Hsinchu, Taiwan

L. Xing

Department of Electrical and Computer Engineering, University of Massachusetts (UMass), Dartmouth, MA, USA

L. Xing

Collaborative Autonomic Computing Laboratory, School of Computer Science and Engineering, University of Electronics Science and Technology of China, Chengdu, China
} 
The first paper in this special issue is on A Circular Polarization Antenna Design for Improving the Reception of UHF RFID Reader, by Jonghun Chun, Hyunsook Chung, and Jongan Park. This paper proposes a novel circular polarization antenna that can improve the reception parameter of a UHF bandwidth RFID reader, and is expected to be extensively used in recognizing multi-tag in the distance.

The second paper in this special issue is on The Biometric based Convertible Undeniable Multi-Signature Scheme to Ensure Multi-Author Copyrights and Profits, by SungHyun Yun, Heuiseok Lim, Young-Sik Jeong, Soon Young Jung, and Jae-Khun Chang. This paper proposes a biometric-based, convertible, and undeniable multi-signature scheme in order to reproduce and manipulate digital content.

The third paper is on Power-efficient M-ary PSSK communications with uncertain phase errors, by Dong Kyoo Kim and Yang Sun Lee. This paper discusses the phase tracking problem of the $M$-ary phase-silence-shift-keying (PSSK) with phase-locked-loop (PLL). Using the analysis result of capacity of the PSSK for the additive white Gaussian noise (AWGN) channel and Rayleigh fading channel, the authors show that the PSSK demodulator can be practically implemented.

The fourth paper in this special issue is on Wireless LAN Access Point Placement Based on User Mobility, by Muhammad Taufiq N., Mohd Fikri Azli bin Abdullah, Deokjai Choi. They suggest a novel WLAN AP placement technique that takes user mobility into consideration.

The fifth paper is on Multi-Path Routing Protocol with Unavailable Areas Identification in Wireless Sensor Networks by Deyun Gao, Oliver Yang, Hongke Zhang, Han-Chie Chao. They propose a new micro sensor multi-path routing protocol (MSMRP) to avoid the unavailable areas when constructing the routes. A neighbor node table exchanging mechanism in MSMRP is used to avoid the multiple paths intersection and to build a new route around the unavailable areas.

The sixth paper is on Centralized Key Management Scheme in Wireless Sensor Networks, by Saber Banihashemian, Abbas Ghaemi Bafghi, Mohammad Hossien Yaghmaee. They present a key management scheme that is more efficient than existing ones in terms of security and connectivity.

The seventh paper is on The impacts of an electronic marketplace with multiple independent retailers for smart grocery ordering systems, by Loo Hay Lee, Chulung Lee, Jie Bao. They investigate the impacts of an electronic marketplace with multiple independent retailers for a smart grocery ordering system and propose both static and dynamic pricing models for the electronic marketplace.

The eighth paper in this special issue is on Ontology based Resource Usage Policy Matching in Computational Grid for Pervasive Computing Applications, by Balchandar R. A., Jong Hyuk Park, Surendran D, Kousalya G. This paper proposes a mechanism for expressing resource usage policies in computational grid for heterogeneous device with varying resource requests.

The ninth paper is on Motion Sequence-based Human Abnormality Detection Scheme for Smart Spaces, by Yoon-Sik Tak, Seungmin Rho, Eenjun Hwang. This paper suggests a new human abnormality detection scheme for surveillance purposes based on human motion, which is one of the most important clues used in accessing a situation for surveillance.

The tenth paper is on A Polygonal Method for Ranging-Based Localization in an Indoor Wireless Sensor Network, by Jaesung Park, Yujin Lim, Kilhung Lee, and Yong-Hoon Choi. They propose an indoor localization method in a wireless sensor network based on IEEE 802.15.4 specification by following a ranging-based approach using not only the measurements of received signal strength (RSS) but also the coordinates of the anchor points (APs). 
The eleventh paper is on Performance of Efficient Signal Detection for LED-ID Systems, by In Hwan Park, Yoon Hyun Kim, Jae Sang Cha, Yeong Min Jang, and Jin Young Kim. They investigate the effects of reader-to-reader interference for LED identification (LED-ID) system in a multi-reader environment, introduce a reader-to-reader interference scenario, derive nominal interrogation range of a desired reader, and evaluate the proposed model.

The twelfth paper is on Tunnel Restraint to Prevent Out-of-Order Packets for Route Optimization in Proxy Mobile IPv6, by Jong-Hyouk Lee, Yeong-Deok Kim, and Dongwoo Lee. They introduce a tunnel restraint scheme to minimize out-of-order packets during a mobile node (MN)'s handover by utilizing a developed estimation function that calculates an amount of out-of-order packets in the RO procedure.

The thirteenth paper in this special issue is on Routing Protocol for Heterogeneous Hierarchical Wireless Multimedia Sensor Networks, by Jin Myoung Kim, Hee Suk Seo, and Jin Kwak. They present a hierarchical heterogeneous network model based routing protocol for wireless multimedia sensor networks (WMSN) in order to support resource constraints, specific QoS, high bandwidth and so on.

The last paper in this special issue is on Energy Efficient Distributed Face Recognition in Wireless Sensor Network, by M. I. Razzak, M. K. Khan, K Alghathbar, J. H. Park. This paper proposes a collaborative face recognition system in wireless sensor networks. Distributed face recognition not only helps to reduce the communication overload but it also increases the node life time by distributing the work load on the nodes

Finally, our special thanks go to Prof. Ramjee Prasad and all editorial staffs for their valuable supports throughout the preparation and publication of this Special Issue. We would like to thank all authors for their contributions to this special issue. We also extend our thanks to all the external reviewers for their great help in reviewing the manuscripts.

\section{Author Biographies}

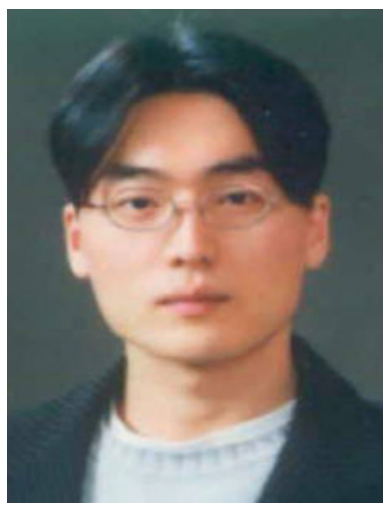

Taeshik Shon received his Ph.D. degree in Information Security from Korea University, Seoul, Korea and his M.S. and B.S. degree in computer engineering from Ajou University, Suwon, Korea. While he was working toward his Ph.D. degree, he was awarded a KOSEF scholarship to be a research scholar in the Digital Technology Center, University of Minnesota, Minneapolis, USA, from February 2004 to February 2005. From Aug. 2005 to Feb. 2011, Dr. Shon had been a senior engineer in the Convergence S/W Lab, DMC R\&D Center of Samsung Electronics Co., Ltd. He is currently a professor at the Division of Information and Computer Engineering, College of Information Technology, Ajou University, Suwon 443-749, Korea. He was awarded the Gold Prize for the Sixth Information Security Best Paper Award from the Korea Information Security Agency in 2003, the Honorable Prize for the 24th Student Best Paper Award from Microsoft-KISS, 2005, the Bronze Prize for the Samsung Best Paper Award, 2006, and the Second Level of TRIZ Specialist certification in compliance with the International TRIZ Association requirements, 2008. He is also serving as an editorial staff and review committee of the Journal of The Korea Institute of Information Security and Cryptology, IAENG International Journal of Computer Science, and other journals. His research interests include Mobile/Wireless Network Security, WPAN/WSN Network Security, network intrusion detection systems, and machine learning. 


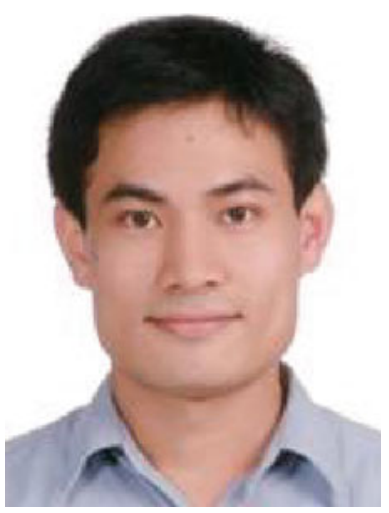

Ching-Hsien Hsu received the B.S. and Ph.D. degrees in Computer Science from Tung Hai University and Feng Chia University, Taiwan, in 1995 and 1999, respectively. He is currently a professor of the department of Computer Science and Information Engineering at Chung Hua University, Taiwan. Dr. Hsu's research interest is primarily in parallel and distributed computing, cloud and grid computing, P2P computing, pervasive computing, services computing, RFID and smart homes. He has published more than 150 academic papers in journals, books and conference proceedings. Dr. Hsu is serving in a number of journal editorial boards, including International Journal of Communication Systems (Wiley), Journal of Computational Science (Elsevier), International Journal of Grid and High Performance Computing (IGI Global), International Journal of Communication Networks and Distributed Systems (Inderscience), Journal of Emerging Technologies in Web Intelligence (Academy Publisher), etc. He has also edited a number of international journal special issues as a guest editor, such as IEEE Transactions on Services Computing, Future Generation Computer Systems, Journal of Supercomputing, Concurrency and Computation: Practice and Experience, The Knowledge Engineering Review, Internet Research, Information System Frontiers. Dr. Hsu has directed and participated in many research projects funded by National Science Council, Ministry of Education, National Center for High Performance Computing and Chung Hua University. Dr. Hsu has severed many international conferences as steering committee, advisory committee, various chairs and committee members. He is a member of Phi Tau Phi Scholastic honor society; IEEE senior member; regional director of the Future Technology Research Association (FTRA); executive committee of IEEE Technical Committee on Scalable Computing (TCSC) and the executive committee of Taiwan Associaton of Cloud Computing (TACC). He was awarded 4 times annual outstanding research award in 2005, 2006, 2007 and 2010, respectively and a distinguished award in 2008 for excellence in research from Chung Hua University.

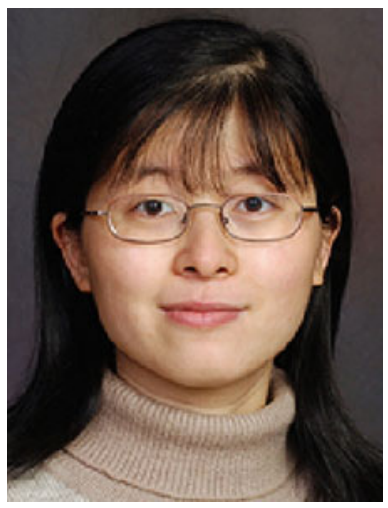

Liudong Xing is an Associate Professor with the Department of Electrical and Computer Engineering at the University of Massachusetts (UMass) Dartmouth. She is also an Adjunct Professor of the Collaborative Autonomic Computing Laboratory at the School of Computer Science and Engineering, University of Electronics Science and Technology of China, Chengdu, China. She received both her M.S. and Ph.D. degrees in electrical engineering from the University of Virginia, Charlottesville. She is the Editor for Short Communications in the International Journal of Performability Engineering. She is also an Associate Editor for the International Journal of Systems Science since 2011. Dr. Xing is the recipient of the Scholar of the Year Award of UMass Dartmouth in 2010 and the IEEE Region 1 Technological Innovation (Academic) Award in 2007. She is also the co-recipient of the Best Paper Award at the IEEE International Conference on Networking, Architecture, and Storage in 2009. Her current research focuses on reliability analysis of complex systems and networks using combinatorial models. She is a senior member of IEEE and a member of Eta Kappa $\mathrm{Nu}$. 